\title{
CONTRIBUIÇÃO AO ESTUDO DAS VARIAÇÕES ANATÔMICAS NA FORMAÇÃO DO SISTEMA PORTAL
}

\author{
CONTRIBUTION TO THE STUDY OF ANATOMICAL VARIATIONS IN PORTAL SYSTEM FORMATION
}

Pedro Duques ${ }^{1}$; Renata S.A. Araújo'; Elivaldo S. de Tolêdo² \& Marcelo F. Rangel ${ }^{3}$

Aluno da Graduação do Curso de Medicina'; Docente - Disciplina de Anatomia Humana do Departamento de Morfologia². Docente Disciplina de $2^{\mathrm{a}}$ Clínica Cirúrgica do Departamento de Cirurgia. Centro de Ciências da Saúde. Universidade Federal da Paraíba Correspondência: Pedro Duques. Av. Argemiro de Figueiredo, 3701/102, Bessa. 58036-030. João Pessoa - PB.Telefone: (83)246-7707/ FAX: (83): 244-8080.

DUQUES P; ARAÚJO RSA; TOLÊDO ES \& RANGEL MF. Contribuição ao estudo das variações anatômicas na formação do sistema portal. Medicina, Ribeirão Preto, 33:486-489, out./dez.2000.

RESUMO: O sistema portal é constituído por uma rede de veias que drenam sangue de grande parte do sistema digestivo para o fígado. Segundo a clássica descrição, a veia porta é formada pela união das veias mesentérica superior e esplênica. A veia mesentérica inferior desemboca na veia esplênica. As variações anatômicas das veias que formam o sistema são freqüentes e os dados da literatura, conflitantes. O objetivo do presente estudo é avaliar a formação do sistema portal em cadáveres e comparar os resultados com os da literatura. Dos 56 cadáveres analisados, $75 \%(n=42)$ apresentavam-se segundo a clássica descrição e $25 \%(n=14)$ apresentavam algum tipo de variação. Destes, $21,4 \%(n=12)$ correspondiam à junção da veia mesentérica inferior com a mesentérica superior e $3,6 \%(n=2)$ à união das veia mesentérica inferior, no ponto de junção das veias esplênica e mesentérica superior, para formar a veia porta. Conclui-se que a situação mais freqüente é a desembocadura da veia mesentérica inferior na veia esplênica. A principal variação encontrada é a desembocadura da veia mesentérica inferior na superior, sendo a confluência das três veias para formar a veia porta uma variação pouco freqüente.

UNITERMOS: Sistema Portal. Anatomia. Veias Mesentéricas. Veia Portal.

\section{INTRODUÇÃO}

O sistema portal é constituído pelas veias que drenam a porção abdominal do tubo digestivo, baço, pâncreas, vesícula biliar e porção distal do esôfago, exceto a parte inferior do canal anal ${ }^{(1)}$.

O principal vaso desse sistema é a veia porta, sendo formada mais freqüentemente pela junção das veias mesentérica superior (VMS) e veia esplênica (VE), segundo relatos na literatura ${ }^{(1 / 11)}$.

A veia porta e suas afluentes originam-se a partir das veias vitelinas do embrião humano. Estas veias drenam plexos capilares desenvolvidos no mesênquima esplancnopleural do saco vitelino. Os recessos superiores do saco vitelino formam o tubo digestivo esplancnopleural, que se estende da membrana orofaríngea à membrana cloacal, onde derivados de todos estes níveis possuem uma drenagem venosa, de origem nas veias vitelinas. Os ramos direito e esquerdo da veia porta são formados a partir dos terminais hepáticos das porções duodenais, direita e esquerda, das veias vitelinas. Devido ao processo de rotação normal do intestino, durante o desenvolvimento, e conseqüente formação da alça duodenal, ocorre atrofia dos segmentos das veias vitelinas originais e da sua anastomose transversal caudal. Por outro lado, 
novos canais venosos surgem para se unir à extremidade esquerda da anastomose intermediária dorsal entre as veias vitelinas, para formar a VMS e a VE. Posteriormente, as outras radículas e raízes principais da veia porta se formam, incluindo a veia mesentérica inferior $(\mathrm{VMI})^{(12)}$.

A veia porta tem comprimento de aproximadamente $8 \mathrm{~cm}$, iniciando-se ao nível da segunda vértebra lombar ${ }^{(1)}$. O sangue colhido das vísceras que drenam para o sistema portal atinge o fígado através desta veia. No interior do fígado, este vaso ramifica-se, terminando em sinusóides, local a partir do qual o sangue novamente converge para alcançar a veia cava inferior, por meio das veias hepáticas ${ }^{(8)}$.

A VMS drena o sangue do intestino delgado, do ceco e dos cólons ascendente e transverso, enquanto a VMI drena o sangue dos cólons sigmóide e descendente e do reto. A VE recolhe o sangue do baço $^{(1)}$.

As variações anatômicas dos vasos que formam o sistema portal são frequientes, e os dados da literatura conflitantes. O presente estudo tem como objetivo avaliar a formação do sistema portal em cadáveres do Setor de Anatomia Humana do Departamento de Morfologia da Universidade Federal da Paraíba, e confrontar os resultados com os da literatura pertinente.

\section{MATERIAL E MÉTODOS}

Foram analisados 56 cadáveres do Setor de Anatomia Humana, Departamento de Morfologia da Universidade Federal da Paraíba, no período de setembro a outubro de 1999. Incluíram-se todos os cadáveres em bom estado de conservação, que continham as principais veias: VMS, VMI, VE e veia porta. Quando necessário, foi realizada a dissecação das peças anatômicas para melhor visualização e definição da condição dos vasos estudados. Excluímos todas as peças que, devido ao estado de conservação, não permitiam o estudo da formação da veia porta. Todas as variações encontradas foram documentadas fotograficamente. Realizou-se revisão da literatura, objetivando comparação com os nossos achados.

\section{RESULTADOS}

De um total de 56 cadáveres analisados, $75 \%$ $(n=42)$ apresentavam-se segundo a clássica descrição anatômica da formação da veia porta composta pela união das VMS e VE, além da VMI desembocando na VE. Vinte e cinco por cento $(n=14)$ das peças anatômicas analisadas apresentavam variações, sendo a mais encontrada $(21,4 \%, \mathrm{n}=12)$ a junção da VMI à VMS (Figura 1). A variação menos freqüente $(3,6 \%$, $\mathrm{n}=02$ ) foi a união da VMI à junção das VE e VMS para formar a veia porta, sendo, portanto, o tronco formador da veia porta constituído por três tributárias.

\section{DISCUSSÃO}

As principais descrições das variações anatômicas do sistema portal, relatadas na literatura, mostram dados conflitantes, tomando-se como exemplo a veia porta formada pela confluência das VMS, VMI e VE, variando de $3 \%$ a 32,6\% dos casos (Tabela I).

A VMI pode unir-se tanto à VMS, em diferentes alturas de seu trajeto, como também à junção das VMS e VE. Dângelo \& Fattini, 1997 ${ }^{(2)}$, relataram que a veia porta pode ser quadrirradicular, quando a veia gástrica esquerda também participa de sua formação.

Abdalla, 1976 $6^{(13)}$, em trabalho utilizando esplenoportografia de pacientes portadores de hipertensão portal, fez observações quanto às variações da desembocadura da VMI, comparando com outros estudos realizados em cadáveres (Tabela I). A casuística de Walcker, $1922^{(14)}$, é a maior delas, onde, dos 165 casos estudados, $42 \%$ apresentavam a VMI desembocando na VE. Em 29\% dos casos a VMI desembocava na VMS, enquanto um menor número (20\%) representava a formação da veia porta pela confluência das VMS, VMI e VE. Em apenas um dos estudos mencionados na Tabela I, com casuística de 100 casos, Purcell et al., 1951(15), observou que em 53\% das vezes a VMI desembocava na VMS contra $28 \%$ dos casos em que a VMI desembocava na VE. Quanto à desembocadura da VMI na VMS, foi reportada uma variação de $18,4 \%$ a $58,7 \%$, enquanto a desembocadura da VMI na VE variou de $28 \%$ a $71 \%$.

Em nosso estudo, dos 56 cadáveres analisados, $75 \%(n=42)$ apresentavam a desembocadura da VMI na VE, sendo a disposição mais freqüente. A principal variação encontrada foi a desembocadura da VMI na VMS, em 21,4\% $(n=12)$. A veia porta trirradicular, formada pela união das VMS, VMI e VE, foi encontrada em poucos casos, 3,6\% ( $\mathrm{n}=2)$. Neste estudo, não foi documentada a veia porta quadrirradicular, formada pela união das veias gástrica esquerda, VMS, VMI e VE(2). 


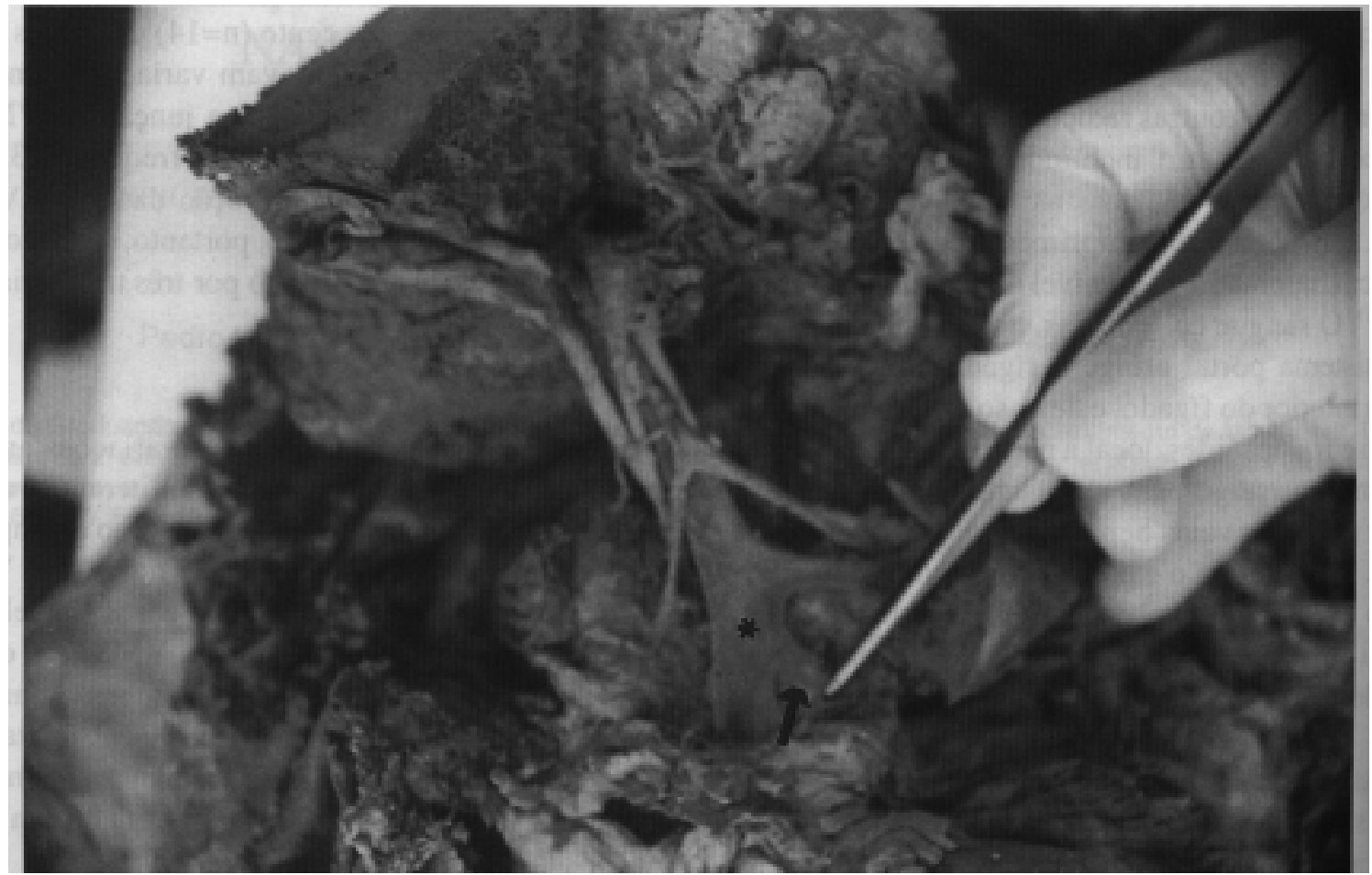

Figura 1: Variação anatômica mais freqüente: veia mesentérica inferior (seta) desembocando na veia mesentérica superior (asterisco).

Tabela I - Estudos das variações anatômicas da veia porta, relatados em sete publicações, acrescidos dos resultados do nosso estudo.

\begin{tabular}{|c|c|c|c|}
\hline Estudos & $\begin{array}{c}\text { Desembocadura } \\
\text { da VMI na VE (\%) }\end{array}$ & $\begin{array}{l}\text { Desembocadura da } \\
\text { VMI na VMS (\%) }\end{array}$ & $\begin{array}{c}\text { Confluência das } \\
\text { VMS, VMI e VE (\%) }\end{array}$ \\
\hline $\begin{array}{l}\text { Walcker( } 1922) \\
165 \text { casos }\end{array}$ & 42 & 29 & 20 \\
\hline $\begin{array}{l}\text { Almeida (1948) } \\
63 \text { casos }\end{array}$ & - & 58,7 & 11,1 \\
\hline $\begin{array}{c}\text { Douglass (1949) } \\
92 \text { casos }\end{array}$ & 38 & 29,8 & 32,6 \\
\hline $\begin{array}{l}\text { Gilfillan (1950) } \\
61 \text { casos }\end{array}$ & 55 & 29,6 & 14,8 \\
\hline $\begin{array}{l}\text { Purcell (1951) } \\
100 \text { casos }\end{array}$ & 28 & 53 & 3,0 \\
\hline $\begin{array}{l}\text { Kelner }(1953) \\
30 \text { casos }\end{array}$ & 50 & 33 & 16,6 \\
\hline $\begin{array}{l}\text { Abdalla (1976) } \\
76 \text { casos }\end{array}$ & 71 & 18,4 & 10,5 \\
\hline $\begin{array}{l}\text { Esta série } \\
56 \text { casos }\end{array}$ & 75 & 21,4 & 3,6 \\
\hline
\end{tabular}


Conclui-se que:

1) a situação anatômica mais freqüentemente encontrada é a desembocadura da VMI na VE e que a principal variação na formação do sistema portal é a desembocadura da VMI na VMS, podendo aquela unirse em diferentes alturas da VMS;

2) no nosso material, a veia porta trirradicular representou uma variação pouco freqüente.

DUQUES P; ARAÚJO RSA; TOLÊDO ES \& RANGEL MF. Contribution to the study of anatomical variations in portal system formation. Medicina, Ribeirão Preto, 33: 486-489, oct./dec.2000.

ABSTRACTS: The portal system is formed by veins which drain the blood from a large part of the digestive system to the liver. According to the classic description, the portal vein is formed by the junction of the superior mesenteric vein and the splenic vein. The inferior mesenteric vein runs into the splenic vein. The anatomical variations of the veins are frequent and the literature data are conflicting. The purpose of this study is to evaluate the portal system in cadaver and compare the results with the ones from the literature. From the 56 cadavers that were analised, $75 \%(n=42)$ presented the classic description and $25 \%(n=14)$ presented some variation. Among them, $21 \%$ $(n=12)$ corresponded to the junction of the inferior mesenteric vein with the superior mesenteric vein and 3,6\% $(n=2)$ showes the union of the inferior mesenteric vein in the junction point of the splenic and superior mesenteric veins in order to form the portal vein. We can conclude that the most frequent situation is the junction of the inferior mesenteric vein with the splenic vein. The main variation in this study was the junction of the inferior mesenteric vein with the superior and the confluence of the three veins in portal vein formation represents a less frequent variation.

UNITERMS: Portal System. Anatomy. Mesenteric Veins. Portal Vein.

\section{REFERÊNCIAS BIBLIOGRÁFICAS}

1 - GRAY HFRS \& GOSS CM. As veias do sistema porta. In: GOSS CM, ed. Gray anatomia, $29^{a}$ ed, Guanabara Koogan, Rio de Janeiro, p 589-591, 1988.

2 - DÂNGELO JG \& FATTINI CM. O abdome. In: DÂNGELO JG \& FATTINI CM, eds. Anatomia humana sistêmica e segmentar, $2^{\text {a }}$ ed, Atheneu, São Paulo, p. 609-610, 1997.

3 - ERHART EA. Esplancnologia. In: ERHART EA, ed. Elementos de anatomia humana, $8^{3}$ ed, Atheneu, São Paulo, p. 239-243, 1992.

4 - GARDNER E; GRAY DJ \& O'RAHILLY R. Fígado, vias biliares, pâncreas e baço. In: GARDNER E, GRAY DJ \& O'RAHILLY R, eds. Anatomia. Estudo regional do corpo humano, 4 ed, Guanabara Koogan, Rio de Janeiro, p. 385-389, 1988.

5 - HOLLINSHEAD WH. O abdome. In: HOLLINSHEAD WH, ed. Anatomia humana, Harper \& Row do Brasil, São Paulo, p. 618-619, 1980.

6 - HOLLINSHEAD WH \& ROSSE C. Intestino e seus derivados. In: HOLLINSHEAD WH \& ROSSE C, eds. Anatomia, $4^{3} \mathrm{ed}$, Interlivros Edições, Rio de Janeiro, p. 557-560, 1985.

7 - LATARJET M \& LIARD AR. Fígado. In: LATARJET M \& LIARD $A R$, eds. Anatomia humana, $2^{3}$ ed, Panamericana, São Paulo, v. 2, p. 1500-1508, 1996.

8 - GRAY HFRS. As veias do abdome e da pelve. In: WILLIAMS PL, WARWICK R, DYSON M \& BANNISTER, eds. Gray anatomia, $37^{\mathrm{a}}$ ed, Guanabara Koogan, Rio de Janeiro, v. 1, p. 796$771,1995$.
9 - SPENCE AP. Sistema digestivo. In: SPENCE AP,ed. Anatomia humana básica, $2^{\mathfrak{a}}$ ed, Manole, São Paulo, p. 558-561, 1991.

10 - EMMANUEL A. Aparelho digestivo. In: EMMANUEL A, ed. Anatomia descritiva, Atheneu, Rio de Janeiro, p. 592595, 1965.

11 - CASTRO SV de. Angiologia. In: CASTRO SV de, ed. Anatomia fundamental, $2^{\mathrm{a}}$ ed, McGraw-Hill do Brasil, São Paulo, p. 265-266, 1985 .

12 - GRAY HFRS. Embriologia. In: WILLIAMS PL, WARWICK R, DYSON M \& BANNISTER, eds. Gray anatomia, $37^{\mathrm{a}} \mathrm{ed}$, Guanabara Koogan, Rio de Janeiro, v. 1, p. 200-204, 1995.

13 - ABDALLA P. Formação do sistema portal. In: Anais, XI Congresso Brasileiro de Anatomia. II Congresso LusoBrasileiro de Anatomia, Niterói-RJ, p. 78-93, 1976.

14 - WALCKER F. Contribution to knowledg of collaterals: the circulation of the blood in the portal system. Langenbeck's Arch Klin Chir 120: 819-858, 1922.

15 - PURCELL HK; CONNOR JJ; ALEXANDER WF \& SCULLY NM. Obeservations on the major radicals of extrahepatic portal system. Arch Surg 62: 670-677, 1951.

Recebido para publicação em 30/03/2000

Aprovado para publicação em 09/08/2000 